Pesq. Vet. Bras. 35(3):249-257, março 2015 DOI: $10.1590 / \mathrm{S} 0100-736 \mathrm{X} 2015000300007$

\title{
Perfil mineral de vacas mestiças Girolanda no período de transição em sistema semi-intensivo em duas estações do ano ${ }^{1}$
}

\author{
Tiago F. Moreira ${ }^{2 *}$, Jose U. Zambrano ${ }^{2}$, Vitor M. de Paula ${ }^{2}$, Fernando P. Casagrande 3 , \\ Elias J. Facury Filho², Lívio R. Molina², Fabíola O.P. Leme ${ }^{2}$ e Antônio U. Carvalho²
}

\begin{abstract}
Moreira T.F., Zambrano J.U., De Paula V.M., Casagrande F.P., Facury Filho E.J., Molina L.R., Leme F.O.P. \& Carvalho A.U. 2015. [Serum mineral profile of Girolanda cows during transition period in semi-intensive system in two seasons.] Perfil mineral de vacas mestiças Girolanda no período de transição em sistema semi-intensivo em duas estações do ano. Pesquisa Veterinária Brasileira 35(3):249-257. Departamento de Clínica e Cirurgia Veterinárias, Escola de Veterinária, Universidade Federal de Minas Gerais, Av. Antônio Carlos 6627, Campus Pampulha, Belo Horizonte, MG 30123-970, Brazil. E-mail: tiago_facury@yahoo.com.br

The metabolic profile of dairy cows in the transition period during summer and winter was evaluated. Were used 31 crossbred Holstein/Gir cows at each season, totaling 62 cows. During summer they remained in pasture with supplementation of corn silage and concentrate and in the winter, corn silage and concentrate was offered to furnish the nutritional requirements. A total of 11 blood samples from each animal were taken according to the following protocol: four collections weekly antepartum, at birth and at 2, 5, 10, 15, 21 and 30 days postpartum. Concentrations of calcium, phosphorus and magnesium were evaluated. All analytes varied according to the physiological status. The average concentrations of calcium and magnesium were higher in summer than in winter. In both seasons, the lowest mean calcium concentrations occurred near calving, and $75 \%$ of the animals in winter and $35.48 \%$ of the animals in the summer were hypocalcemic. Only on the tenth day postpartum, the calcium concentrations returned to levels of prepartum, demonstrating that this time is necessary for the adaptation of the new condition of lactating. Magnesium concentrations were lower postpartum than prepartum in both seasons, demonstrating the need for this mineral for the production of milk. The average concentrations of magnesium remained always within the reference values for the species, but $19 \%$ of the animals at two days postpartum in winter and $7 \%$ of the animals at 10 days postpartum in summer had concentrations below these limits. Phosphorus concentrations suffered large variation along the peri-partum, but always at high levels, with $49.8 \%$ of the animals in winter and $37.3 \%$ in summer had phosphorus levels above the reference range of the species.
\end{abstract}

INDEX TERMS: Mineral profile, hypocalcemia, hypomagnesemia, peripartum, monitoring, dairy cattle.

RESUMO-- Neste estudo foi avaliado o perfil metabólico de vacas leiteiras no período de transição durante o verão e o inverno. Foram utilizados 31 animais pluríparos mestiços

\footnotetext{
${ }^{1}$ Recebido em 7 de agosto de 2014.

Aceito para publicação em 18 de março de 2015.

${ }^{2}$ Departamento de Clínica e Cirurgia Veterinárias, Escola de Veterinária, Universidade Federal de Minas Gerais (UFMG), Av. Antônio Carlos 6627, Cx. Postal 567, Campus Pampulha, Belo Horizonte, MG 30123-970, Brasil. *Autor para correspondência: tiago_facury@yahoo.com.br

${ }^{3}$ Departamento de Anatomia, Patologia e Clínicas Veterinárias, Escola de Medicina Veterinária e Zootecnia, Universidade Federal da Bahia, Av. Adhemar de Barros 500, Campus Ondina, Salvador, BA 40170-110, Brasil.
}

girolando em cada estação, totalizando 62 vacas. No verão permaneciam em pasto com suplementação de silagem de milho e concentrado. No inverno, a exigência nutricional era suprida apenas com silagem de milho e concentrado. Foram feitas um total de 11 coletas de sangue de cada animal segundo o seguinte protocolo: quatro coletas pré-parto espaçadas semanalmente, no dia do parto e com 2, 5, 10, 15, 21 e 30 dias pós-parto. Foram avaliadas as concentrações de cálcio, fósforo e magnésio. Todos os analitos variaram em função do estatus fisiológico. As concentrações médias de cálcio e magnésio foram maiores no verão do que no inverno. Em ambas as estações, as menores concentra- 
ções médias de cálcio ocorreram próximas ao parto, sendo que $75 \%$ dos animais no inverno e $35,48 \%$ dos animais no verão estavam hipocalcêmicos. Apenas no décimo dia as concentrações de cálcio voltaram aos níveis do pré-parto, demonstrando que esse tempo é necessário para a adaptação da nova condição de lactante. As concentrações de magnésio foram menores no pós-parto do que no pré-parto nas duas estações, demonstrando a necessidade desse mineral para a produção de leite. As concentrações médias de magnésio permaneceram sempre dentro dos valores de referência, porém 19\% dos animais aos dois dias pós-parto no inverno e 7\% dos animais aos 10 dias pós-parto no verão tinham concentrações inferiores a esses limites. As concentrações de fósforo sofreram grande variação ao longo do peri-parto, porém sempre em níveis elevados, sendo que $49,8 \%$ dos animais no inverno e $37,3 \%$ no verão tinham níveis de fósforo acima dos valores de referência.

TERMOS DE INDEXAÇÃO: Perfil mineral, hipocalcemia, hipomagnesemia, periparto, monitoramento, gado de leite.

\section{INTRODUÇÃO}

Os minerais são essenciais para qualquer organismo atingir a homeostase, o equilíbrio necessário para viver, mas sofrem grandes alterações em seus níveis e em seu metabolismo durante o período do periparto (NRC 2001, Drackley 2005). Nesse período, a homeostase mineral passa por grande desafio, se destacando os macrominerais cálcio (Ca), magnésio ( $\mathrm{Mg}$ ) e fósforo (P). Isto ocorre principalmente devido à súbita necessidade desses minerais para o desenvolvimento do feto e para a produção de colostro e leite pela vaca (Horst et al. 1997, Drackley 2005, DeGaris \& Lean 2008, Goff 2008, Froetschel 2011, Oetzel 2013).

Apesar da necessidade destes minerais aumentarem no periparto, o animal diminui o seu consumo de alimento durante este período (Drackley 2005). Neste cenário, alguns minerais sofrem uma queda a níveis abaixo do fisiológico em suas concentrações no final de gestação e início da lactação (Goff, 2006) e, dessa forma, as vacas podem apresentar quadros de hipocalcemia, hipomagnesemia e hipofosfatemia.

A concentração sérica normal de cálcio no bovino adulto se mantém em torno de 8,5 a $10 \mathrm{mg} / \mathrm{dL}$ sendo que $45 \mathrm{a}$ $50 \%$ estão na forma livre (Littledike \& Goff 1987, Larsen 2001, Goff 2004). A variação da concentração de cálcio, principalmente na forma ionizada, não deve fugir deste valor, do contrário ocorrem várias consequências graves para os processos fisiológicos e para a vida, como a perda do potencial elétrico e condutividade de membrana de músculos e nervos (Littledike \& Goff 1987, NRC 2001).

Quando o nível de cálcio circulante é reduzido, a vaca pode apresentar a forma clínica da hipocalcemia, caracterizada por disfunção neuromuscular, paralisia flácida e depressão da consciência (Goff 2008, Oetzel \& Goff 2009, Oetzel 2013). Porém, não é apenas quando o animal apresenta sinais clínicos que a hipocalcemia pode prejudicá-lo. A queda dos níveis plasmáticos de cálcio abaixo dos valores fisiológicos sem a manifestação clínica é chamada de hipocalcemia subclínica e também traz graves consequências. Pode levar a diminuição da motilidade do rumem e abo- maso, diminuição da ingestão de alimentos, inibição da secreção de insulina, e diminuição da resposta imunológica (Goff \& Horst 1997b, Larsen 2001, Kimura et al. 2006, Oetzel \& Goff 2009, Reinhardt et al. 2011, Oetzel 2013). Como consequência de todas essas alterações ocorre aumento da incidência de distocia, deslocamento de abomaso, mastite, prolapso uterino, cetose, diminuição da produção de leite e esses animais acabam sendo descartados mais prematuramente (Horst et al. 1997, Goff \& Horst 1997b, Larsen 2001, Melendez et al. 2004, Mulligan et al. 2006, Kimura et al. 2006, Goff 2008, Chapinal et al. 2011, Reinhardt et al. 2011, Roberts et al. 2012, Oetzel 2013).

Desempenhando um papel tão importante para determinar a saúde da vaca no início de lactação, o diagnóstico da hipocalcemia subclínica é de extrema importância; porém, o quadro é de difícil diagnóstico apenas com exames de rotina nas fazendas, necessitando de exame laboratorial de bioquímica sanguínea (Oetzel 2004, Goff 2008).

0 uso do perfil metabólico começou em 1960 (Payne et al. 1970), porém foi no final dos anos 70 que a técnica foi utilizada como parte de programas de monitoramento de saúde e produtividade dos rebanhos (Whitaker et al. 1999). A partir de então essa técnica é utilizada amplamente em vários países, principalmente europeus (Whitaker 2004, Stengärde 2010).

Este trabalho foi desenvolvido com o objetivo de comparar e descrever o comportamento da concentração sérica de cálcio, fósforo e magnésio e a ocorrência de distúrbios metabólicos relacionados a esses minerais no período de transição de vacas leiteiras, em sistema semi-intensivo em duas épocas do ano (verão e inverno), as quais apresentam condições climáticas e de manejo distintas.

\section{MATERIAL E MÉTODOS}

O experimento foi aprovado pelo Comitê de Ética em Experimentação Animal (CETEA/UFMG) sobre o protocolo de número 82/2011.

A pesquisa foi realizada em 2011, em dois momentos diferentes, de janeiro a abril (Período 1: Verão) e de maio a agosto (Período 2: Inverno) em uma fazenda localizada no município de Martinho Campos, Minas Gerais. A região possui duas estações climáticas bem definidas: verão quente e chuvoso, e inverno ameno e seco. Segundo a classificação de Köppen, o clima dessa região é classificado como tropical de savana com estação seca de inverno (Aw). As temperaturas média, máxima e mínima e a precipitação durante o experimento podem ser observadas na Figura 1.

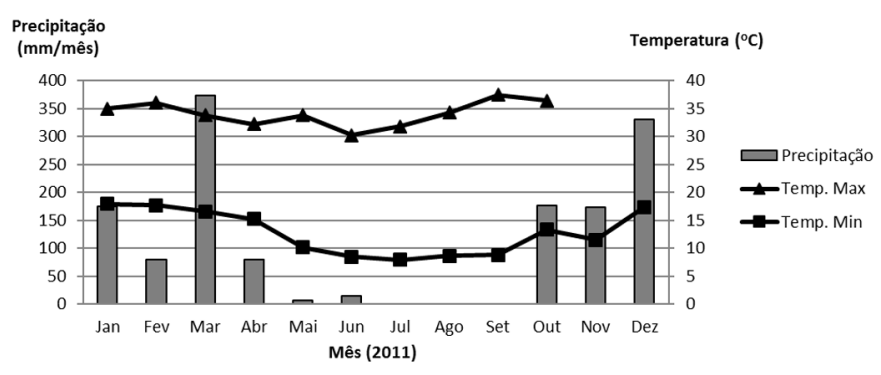

Fig.1. Temperatura ambiental máxima e mínima e pluviosidade durante o ano de 2011 em Pompeu/MG. Dados obtidos da estação meteorológica do Instituto Nacional de Meteorologia (Inmet) de Pompeu. 
A propriedade produzia cerca de 5.000 litros de leite por dia, com 250 a 300 animais em lactação. No experimento foram avaliadas 31 vacas em cada período, totalizando 62 animais. As vacas eram mestiças com graus de sangue variando de 5/8 até 31/32 Holandês-Zebu e com ordem de lactação entre a segunda e a sétima. A média de produção foi de $20 \mathrm{~L} /$ dia no verão e 23L/dia no período de inverno.

As vacas secas permaneciam em um pasto afastado até completarem 30 dias para a data do parto previsto, quando eram transferidas para o piquete maternidade que ficava mais próximo da área de manejo, com aproximadamente cinco hectares formado por Brachiaria brizantha, com áreas de sombra natural e sombrites. A lotação variava entre 25 e 35 animais, entre vacas e novilhas e o alimento era fornecido em cocho de cimento com $8 \mathrm{~m}$ de comprimento aproximadamente.

Durante os meses de janeiro a abril, os animais da propriedade consumiram pasto além de silagem de milho e ração misturados em vagão forrageiro e fornecidos no cocho. Nos meses de maio a agosto, era fornecida dieta total com silagem de milho e ração em quantidade suficiente para suprir as exigências nutricionais. Em ambos os períodos as dietas eram formuladas para suprir todos os requerimentos nutricionais segundo o NRC (2001) (Quadro 1). Em todos os piquetes havia bebedourose cocho de sal mineral. As vacas no pré-parto não recebiam sal aniônico.

A ração fornecida em ambas as estações para as vacas em lactação era composta por milho grão, farelo de soja, bicarbonato de sódio, ureia, fosfato bicálcico, sal, monensina e núcleo vitamínico e mineral, sendo que a proporção dos ingredientes variou pouco.

As coletas de sangue foram realizadas semanalmente no pré-parto, começando na quarta semana anterior a data prevista do parto, depois uma coleta no prazo máximo de 24 h após o parto e, posteriormente, seis coletas, nos dias 2, 5, 10, 15, 21 e 30 pós-parto.

0 sangue foi colhido em tubos a vácuo com gel ativador de coágulo, através da punção da veia coccígea após assepsia do local.

\section{Quadro1. Composição da dieta oferecida no verão (janeiro a abril) e no inverno (maio a agosto) para as vacas em lactação e para o lote pré-parto na propriedade}

\begin{tabular}{|c|c|c|c|c|}
\hline & \multirow[t]{2}{*}{ Unidade } & \multicolumn{2}{|c|}{ Lote pós-parto } & \multirow[t]{2}{*}{ Lote pré-parto } \\
\hline & & Verão & Inverno & \\
\hline CMS & kg MS/Dia & 18,6 & 20,5 & 11,5 \\
\hline $\mathrm{PB}$ & \%MS & 17,5 & 16 & 14,5 \\
\hline ENERGIA LIQ. & Mcal/kgMS & 1,63 & 1,6 & 1,55 \\
\hline NDT & $\%$ & 72,4 & 70,9 & 69,7 \\
\hline FDN & $\%$ & 31,5 & 31,4 & 40,6 \\
\hline FDA & $\%$ & 17,7 & 21,3 & 23 \\
\hline CNF & $\%$ & 41,4 & 44,4 & 36,4 \\
\hline AMIDO & $\%$ & 34 & 21,9 & 28,4 \\
\hline LIPIDEO & $\%$ & 2,8 & 3,3 & 2,8 \\
\hline CINZAS & $\%$ & 7,4 & 6,1 & 6,2 \\
\hline $\mathrm{Ca}$ & $\%$ & 0,69 & 0,72 & 0,48 \\
\hline $\mathrm{P}$ & $\%$ & 0,39 & 0,37 & 0,34 \\
\hline $\mathrm{Mg}$ & $\%$ & 0,23 & 0,21 & 0,3 \\
\hline $\mathrm{K}$ & $\%$ & 1,14 & 0,85 & 1,18 \\
\hline $\mathrm{Na}$ & $\%$ & 0,35 & 0,27 & 0,1 \\
\hline $\mathrm{Cl}$ & $\%$ & 0,1 & 0,14 & 0,22 \\
\hline S & $\%$ & 0,19 & 0,15 & 0,18 \\
\hline Co & Ppm & 0,3 & 0,3 & 0,3 \\
\hline $\mathrm{Cu}$ & Ppm & 13 & 10 & 12 \\
\hline I & Ppm & 0,7 & 0,6 & 0,7 \\
\hline $\mathrm{Mn}$ & Ppm & 57 & 44 & 51 \\
\hline $\mathrm{Se}$ & Ppm & 0,37 & 0,28 & 0,33 \\
\hline $\mathrm{Zn}$ & Ppm & 59 & 46 & 51 \\
\hline Vit A & KUI & 5 & 4 & 8 \\
\hline Vit D & KUI & 1,2 & 0,9 & 1,9 \\
\hline Vit E & UI & 29 & 22 & 47 \\
\hline
\end{tabular}

As coletas do pré-parto foram realizadas sempre em um mesmo dia da semana no período da manhã logo antes de ser fornecido o alimento, e as coletas do pós-parto eram feitas após a ordenha da tarde.

0 sangue foi centrifugado e o soro separado em quatro alíquotas de $0,5 \mathrm{ml}$, congeladas $\left(-20^{\circ} \mathrm{C}\right)$ e enviadas para o Laboratório de Patologia Clínica da UFMG para posterior análise.

Foram realizadas dosagens de cálcio, fósforo e magnésio utilizando técnica de espectrofotometria em aparelho automático marca Cobas modelo Mira ${ }^{\circledR}$ e kits comerciais Synermed ${ }^{\circledR}$.

Para comparar o comportamento das variáveis estudadas entre as estações do ano, o delineamento estatístico utilizado foi organizado em Arranjo em Parcelas Subdivididas, no qual as estações do ano representam as parcelas e os momentos das coletas as sub-parcelas. Usou-se o software estatístico SAEG 9.1. As médias entre os momentos de coleta foram comparadas pelo teste Scott-Knott e entre as estações do ano a comparação foi feita através do teste de SNK, e para a análise da condição corporal foi utilizado o teste Mann-Whitney para comparação dos tempos de coleta e o teste de comparação múltipla de Dunn para comparação entre período do ano, todos com 5\% de significância (Sampaio 2010).

\section{RESULTADOS}

Os resultados foram analisados de duas formas: através da concentração média em cada momento (Quadro 2, Fig.2 e 3) e da proporção de animais com concentração fora do intervalo de referência para a espécie (Quadro 3, Fig.4), como foi preconizado por Oetzel (2004).

A estação do ano influenciou a concentração de todos os minerais. As concentrações séricas médias de cálcio e magnésio foram maiores no verão com médias de 9,61mg/dL e $2,88 \mathrm{mg} / \mathrm{dL}$ respectivamente, enquanto no inverno as concentrações foram de $8,95 \mathrm{mg} / \mathrm{dL}$ e $2,61 \mathrm{mg} / \mathrm{dL}$ respectivamente, como demonstrado no Quadro 2. As concentrações de cálcio e magnésio se comportaram de forma semelhante ao longo do período estudado nas duas épocas do ano. A calcemia apresentou queda a partir da primeira semana pré-parto até o dia 10 pós-parto. Depois desta data, aumentou no dia 15 e permaneceu assim até o final das coletas no dia 30 pós-parto (Fig.2).

As concentrações médias de cálcio no dia do parto foram de $9,28 \mathrm{mg} / \mathrm{dL}$ no verão e de $8,13 \mathrm{mg} / \mathrm{dL}$ no inverno, estando este último abaixo dos valores considerados fisio-

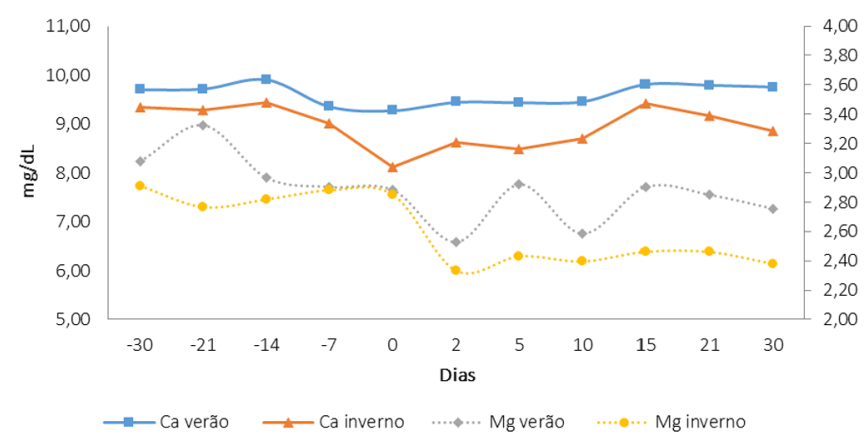

Fig.2. Concentração sérica de cálcio ( $\mathrm{Ca}$, coluna da esquerda) e magnésio ( $\mathrm{Mg}$, coluna da direita) em vacas mestiças pluríparas na quarta ( -4 sem.), terceira ( -3 sem.), segunda ( -2 sem.) e primeira ( -1 sem.) semanas pré-parto, parto e nos dias 2,5 , $10,15,21$ e 30 pós-parto em sistema semi-intensivo no verão e inverno. 
Quadro2. Médias e desvio padrão (DP) das concentrações de cálcio total sérico (mg/dL), magnésio (mg/dL) e fósforo ( $\mathrm{mg} / \mathrm{dL})$ de vacas mestiças pluríparas na quarta $(-4 \mathrm{sem}$.) terceira (-3 sem.), segunda (-2 sem.) e primeira (-1 sem.) semanas pré-parto, parto e nos dias $2,5,10,15$, 21 e 30 pós-partoem sistema semi-intensivo no verão e inverno

\begin{tabular}{|c|c|c|c|c|c|c|c|c|c|c|c|c|c|}
\hline & \multirow{2}{*}{$\begin{array}{r}\text { Estação } \\
\text { do ano }\end{array}$} & \multicolumn{11}{|c|}{ Tempo } & \multirow{2}{*}{ Média } \\
\hline & & $-4 \mathrm{sem}$. & -3 sem. & -2 sem. & -1 sem. & Parto & 2 dias & 5 dias & 10 dias & 15 dias & 21 dias & 30 dias & \\
\hline \multirow[t]{6}{*}{ Cálcio } & Verão & 9,70 & 9,72 & 9,90 & 9,36 & 9,28 & 9,45 & 9,44 & 9,46 & 9,81 & 9,79 & 9,76 & $9,61^{\mathrm{a}}$ \\
\hline & $D P$ & $+1,18$ & $+1,19$ & $+1,15$ & $+1,37$ & $+1,31$ & $+1,54$ & $+0,78$ & $+1,80$ & $+1,33$ & $+1,22$ & $+1,32$ & $+1,31$ \\
\hline & Inverno & 9,34 & 9,28 & 9,44 & 9,01 & 8,13 & 8,62 & 8,48 & 8,69 & 9,42 & 9,16 & 8,86 & $8,95^{\mathrm{b}}$ \\
\hline & $D P$ & $+0,96$ & $+0,99$ & $+0,81$ & $+0,93$ & $+1,08$ & $+0,93$ & $+1,05$ & $+0,94$ & $+1,34$ & $+1,12$ & $+0,86$ & $+1,07$ \\
\hline & Média & $9,52^{\mathrm{A}}$ & $9,50^{A}$ & $9,67^{A}$ & $9,19^{\mathrm{B}}$ & $8,70^{\mathrm{B}}$ & $9,03^{\mathrm{B}}$ & $8,96^{\mathrm{B}}$ & $9,08^{\mathrm{B}}$ & $9,62^{\mathrm{A}}$ & $9,47^{\mathrm{A}}$ & $9,31^{\mathrm{A}}$ & 9,28 \\
\hline & $D P$ & $+1,07$ & $+1,11$ & $+1,01$ & $+1,18$ & $+1,33$ & $+1,33$ & $+1,04$ & $+1,48$ & $+1,34$ & $+1,20$ & $+1,18$ & $+1,24$ \\
\hline \multirow[t]{6}{*}{ Magnésio } & Verão & 3,0 & 3,33 & 2,97 & 2,90 & 2,88 & 2,53 & 2,92 & 2,59 & 2,90 & 2,85 & 2,76 & $2,88^{a}$ \\
\hline & & $+0,6$ & $+1,03$ & $+0,43$ & $+0,57$ & $+0,49$ & $+0,38$ & $+1,06$ & $+0,61$ & $+0,81$ & $+0,52$ & $+0,47$ & $+0,70$ \\
\hline & Inverno & 2,91 & 2,77 & 2,82 & 2,88 & 2,85 & 2,33 & 2,43 & 2,40 & 2,46 & 2,46 & 2,38 & $2,61^{b}$ \\
\hline & $D P$ & $+0,44$ & $+0,52$ & $+0,56$ & $+0,56$ & $+0,78$ & $+0,78$ & $+1,30$ & $+0,59$ & $+0,45$ & $+0,64$ & $+0,51$ & $+0,72$ \\
\hline & Média & $2,99^{A}$ & $3,05^{\mathrm{A}}$ & $2,89^{A}$ & $2,89^{A}$ & $2,87^{\mathrm{A}}$ & $2,43^{\mathrm{B}}$ & $2,68^{\mathrm{B}}$ & $2,49^{\mathrm{B}}$ & $2,68^{\mathrm{B}}$ & $2,66^{\mathrm{B}}$ & $2,57^{\mathrm{B}}$ & 2,75 \\
\hline & $D P$ & $+0,53$ & $+0,86$ & $+0,50$ & $+0,56$ & $+0,65$ & $+0,63$ & $+1,21$ & $+0,60$ & $+0,67$ & $+0,61$ & $+0,53$ & $+0,72$ \\
\hline \multirow[t]{6}{*}{ Fósforo } & Verão & $9,02^{\mathrm{aA}}$ & $8,12^{\mathrm{aA}}$ & $8,32^{\mathrm{aA}}$ & $7,51^{\mathrm{aB}}$ & $7,21^{\mathrm{aB}}$ & $8,49^{\mathrm{aA}}$ & $7,83^{\mathrm{aB}}$ & $6,95^{\mathrm{aB}}$ & $7,16^{\mathrm{bB}}$ & $7,29^{\mathrm{aB}}$ & $6,95^{\mathrm{aB}}$ & 7,71 \\
\hline & $D P$ & $+1,79$ & $+1,91$ & $+1,89$ & $+1,53$ & $+2,43$ & $+1,72$ & $+2,06$ & $+1,68$ & $+1,47$ & $+2,18$ & $+1,89$ & $+1,96$ \\
\hline & Inverno & $9,11^{\mathrm{aA}}$ & $8,25^{\mathrm{aA}}$ & $8,71^{\mathrm{aA}}$ & $8,29^{\mathrm{aA}}$ & $7,87^{\mathrm{aB}}$ & $7,32^{\mathrm{aB}}$ & $6,60^{\mathrm{aB}}$ & $7,77^{\mathrm{aB}}$ & $8,25^{\mathrm{aA}}$ & $8,57^{\mathrm{aA}}$ & $7,64^{\mathrm{aB}}$ & 8,04 \\
\hline & & $+1,65$ & $+1,09$ & $+1,83$ & $+1,85$ & $+2,19$ & $+2,57$ & $+1,75$ & $+2,46$ & $+2,34$ & $+2,23$ & $+1,75$ & $+2,09$ \\
\hline & Média & 9,06 & 8,18 & 8,52 & 7,90 & 7,54 & 7,90 & 7,21 & 7,36 & 7,71 & 7,93 & 7,30 & 7,87 \\
\hline & $D P$ & $+1,69$ & $+1,54$ & $+1,85$ & $+1,73$ & $+2,32$ & $+2,25$ & $+1,99$ & $+2,12$ & $+2,01$ & $+2,28$ & $+1,84$ & $+2,03$ \\
\hline
\end{tabular}

$\overline{\text { Entre as colunas, a }}$ médias seguidas por letras maiúsculas distintas diferem entre si pelo teste de Scott-Knott $(\mathrm{P}<0,05)$. Entre as linhas, as médias seguidas por letras minúsculas distintas diferem entre si pelo teste $\mathrm{SNK}(\mathrm{P}<0,05)$.

Quadro 3. Incidência (\%) de hipocalcemia (concentração sérica de Ca <8,5 mg /dL; Goff 2004), hipomagnesemia (concentração sérica de $\mathrm{Mg}<1,7 \mathrm{mg} / \mathrm{dL}$; Dalley 1992) e hiperfosfatemia (fósforo sérico >8,0 mg/dL; Goff 2000) de vacas mestiças pluríparas na quarta (-4 sem.) terceira (-3 sem.), segunda (-2 sem.) e primeira (-1 sem.) semanas pré-parto, parto e nos dias $2,5,10,15,21$ e 30 pós-parto em sistema semi-intensivo no verão e inverno

\begin{tabular}{|c|c|c|c|c|c|c|c|c|c|c|c|c|c|c|c|c|c|c|c|c|c|c|}
\hline & \multicolumn{22}{|c|}{ Tempo } \\
\hline & \multicolumn{2}{|c|}{-4 sem. } & \multicolumn{2}{|c|}{-3 sem. } & \multicolumn{2}{|c|}{-2 sem. } & \multicolumn{2}{|c|}{-1 sem. } & \multicolumn{2}{|c|}{ Parto } & \multicolumn{2}{|c|}{2 dias } & \multicolumn{2}{|c|}{5 dias } & \multicolumn{2}{|c|}{10 dias } & \multicolumn{2}{|c|}{15 dias } & \multicolumn{2}{|c|}{21 dias } & \multicolumn{2}{|c|}{30 dias } \\
\hline & Ver & Inv & Ver & Inv & Ver & Inv & Ver & Inv & Ver & Inv & Ver & Inv & Ver & Inv & Ver & Inv & Ver & Inv & Ver & Inv & Ver & Inv \\
\hline Hipocalcemia (\%) & 15 & 13 & 16 & 26 & 16 & 13 & 26 & 39 & 35 & 75 & 26 & 45 & 13 & 52 & 27 & 47 & 14 & 38 & 11 & 23 & 4 & 23 \\
\hline Hiperfosfatemia (\%) & 63 & 75 & 45 & 55 & 52 & 71 & 26 & 57 & 32 & 39 & 58 & 29 & 40 & 13 & 17 & 48 & 21 & 61 & 36 & 63 & 21 & 37 \\
\hline Hipomagnesemia (\%) & 0 & 0 & 0 & 0 & 0 & 0 & 0 & 0 & 0 & 0 & 0 & 19 & 0 & 17 & 7 & 10 & 0 & 4 & 0 & 7 & 0 & 7 \\
\hline
\end{tabular}

lógicos (8,5-10mg/dL segundo Goff 2004). Nesse momento, a incidência de hipocalcemia subclínica foi a mais elevada sendo observada em $35,48 \%$ dos animais no verão e em $75 \%$ no inverno. Em todas as coletas e em ambas as estações existiam animais com níveis séricos de cálcio abaixo do fisiológico, porém não ocorreu nenhum caso de hipocalcemia clínica. Importante ressaltar que mesmo aos 30 dias pós-parto ainda existiam animais hipocalcêmicos, principalmente no período de inverno (23\%) (Quadro 3, Fig.4).

As concentrações médias de magnésio foram menores no pós-parto que no pré-parto. Estas não apresentaram alterações entre a quarta semana pré-parto e o dia do parto, apresentando uma queda em seus níveis a partir de dois dias pós-parto e se mantiveram em níveis similares até o final do experimento.

Durante todos os momentos avaliados, as concentrações médias de magnésio permaneceram dentro dos níveis fisiológicos (1,7-3,3mg/dL, Reinhardt et al. 1988, Dalley 1992), porém alguns animais apresentaram concentrações abaixo destes valores no pós-parto, principalmente no período de inverno. A maior incidência de hipomagnesemia ocorreu no inverno aos dois dias pós-parto, acometendo $19 \%$ dosanimais. No verão, casos de hipomagnesemia ocorreram apenas no dia 10 pós-parto, em 7\% das vacas (Quadro 2, Fig.2).

O comportamento das concentrações séricas de fósforo foi diferente entre as duas estações do ano (Quadro 2), porém, os valores foram similares nos dois períodos, apresentando diferença apenas no dia 15 pós-parto, com a concentração no verão superior a do inverno. No verão a queda das concentrações médias de fósforo ocorreu mais cedo, na

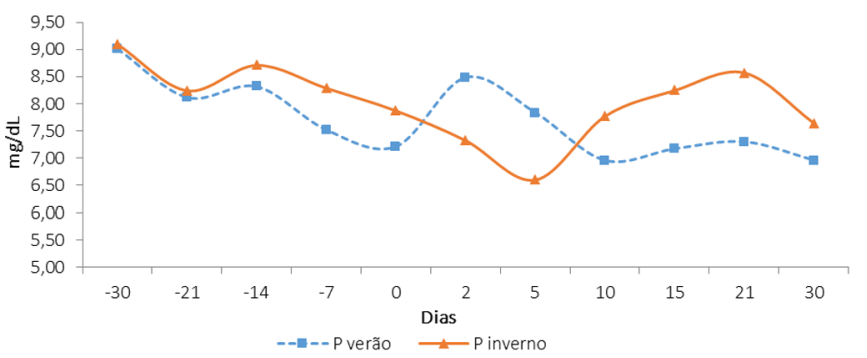

Fig.3. Concentração sérica de fósforo (P) em de vacas mestiças pluríparas na quarta ( $-4 \mathrm{sem}$.) terceira ( $-3 \mathrm{sem}$.), segunda ( -2 sem.) e primeira ( -1 sem.) semanas pré-parto, parto e nos dias $2,5,10,15,21$ e 30 pós-parto em sistema semi-intensivo no verão e inverno. 
Quadro 4. Valores de condição corporal das vacas mestiças pluríparas na quarta (-4 sem.) terceira (-3 sem.), segunda (-2 sem.) e primeira (-1 sem.) semanas pré-parto, parto e nos dias $2,5,10,15,21$ e 30 pós-parto em sistema semi-intensivo em duas estações do ano

\begin{tabular}{ccccccccccccc}
\hline & \multicolumn{10}{c}{ Tempo } \\
\cline { 2 - 12 } Verão & -4 sem. & -3 sem. & -2 sem. & -1 sem. & Parto & 2 dias & 5 dias & 10 dias & 15 dias & 21 dias & 30 dias \\
& Mediana & $3,4^{\mathrm{bABCD}}$ & $3,5^{\mathrm{bAB}}$ & $3,5^{\mathrm{bAB}}$ & $3,5^{\mathrm{bA}}$ & $3,5^{\mathrm{aAB}}$ & $3,5^{\mathrm{aABC}}$ & $3,5^{\mathrm{aABC}}$ & $3,5^{\mathrm{aABC}}$ & $3^{\mathrm{aCBD}}$ & $3^{\mathrm{aD}}$ & $3^{\mathrm{aCD}}$ \\
& Média & 3,40 & 3,40 & 3,42 & 3,45 & 3,40 & 3,35 & 3,35 & 3,28 & 3,16 & 3,07 & 3,07 \\
& Máxima & 4,5 & 4,5 & 4,5 & 4,5 & 4,5 & 4 & 4 & 4 & 4 & 4 & 3,5 \\
Mínima & 3 & 3 & 3 & 3 & 2,5 & 2,5 & 2,5 & 2,5 & 2,5 & 2,5 & 2,5 \\
Inverno & Mediana & $3,7^{\mathrm{aA}}$ & $3,5^{\mathrm{aA}}$ & $3,5^{\mathrm{aA}}$ & $3,5^{\mathrm{aA}}$ & $3,5^{\mathrm{aAB}}$ & $3,5^{\mathrm{aABC}}$ & $3,5^{\mathrm{aBCD}}$ & $3^{\text {aCD }}$ & $3^{\mathrm{aCD}}$ & $3^{\mathrm{aD}}$ & $3^{\mathrm{aD}}$ \\
Média & 3,69 & 3,65 & 3,73 & 3,66 & 3,58 & 3,44 & 3,31 & 3,21 & 3,13 & 3,12 & 3,05 \\
Máxima & 4,5 & 4,5 & 4,5 & 4,5 & 4,5 & 4 & 4 & 4 & 4 & 4 & 4 \\
Mínima & 3 & 3 & 3 & 3 & 3 & 3 & 2,5 & 2,5 & 2,5 & 2,5 & 2,5
\end{tabular}

Entre as colunas, as médias seguidas por letras maiúsculas distintas diferem entre si pelo teste de comparação múltipla de Dunn $(\mathrm{P}<0,05)$. Entre as linhas, as médias seguidas por letras minúsculas distintas diferem entre si pelo teste Mann-Whitney $(\mathrm{P}<0,05)$.

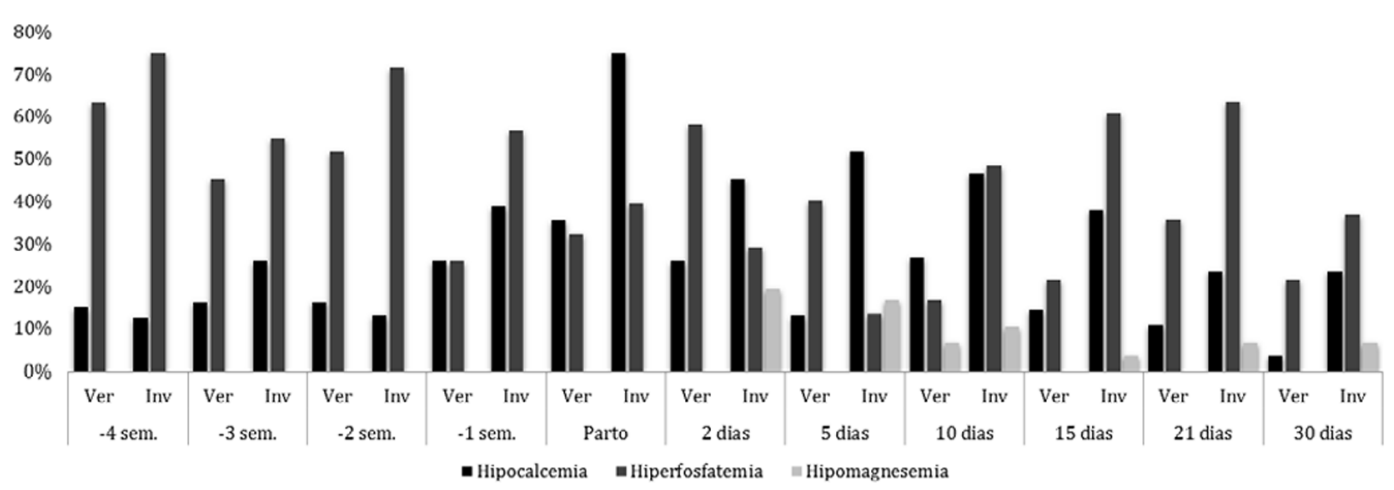

Fig.4. Incidência de hipocalcemia (concentração sérica de Ca <8,5 mg /dL), hipomagnesemia (concentração sérica de Mg <1,7mg/dL; Dalley, 1992) e hiperfosfatemia (fósforo sérico $>8,0 \mathrm{mg} / \mathrm{dL}$; Goff, 2000) de vacas mestiças pluríparas na quarta ( -4 sem.) terceira ( -3 sem.), segunda (-2 sem.) e primeira (-1 sem.) semanas pré-parto, parto e nos dias 2, 5, 10, 15, 21 e 30 pós-parto em sistema semi-intensivo no verão e inverno.

semana anterior ao parto, enquanto no inverno a concentração só apresentou queda no dia do parto (Quadro 2, Fig.3). No verão, aos dois dias pós-parto, as concentrações se recuperaram, mas voltaram a cair logo em seguida e se mantiveram neste nível durante o restante do experimento. No inverno, as concentrações de fósforo só retornaram aos valores similares aos encontrados inicialmente nos dias 15 e 21 pós-parto, porém voltaram a cair no dia 30 pós-parto.

Em nenhum momento a concentração média de fósforo foi menor que os valores de referência de 4 a $8 \mathrm{mg} / \mathrm{dl}$ (Goff 2004), apesar de a concentração estar abaixo desse valor em alguns animais. A maior incidência de hipofosfatemia ocorreu no dia do parto com dois animais no verão $(6,45 \%)$ e um no inverno (3,5\%). As concentrações de fósforo apresentaram outro distúrbio, a hiperfosfatemia, que apresentou grande incidência durante todo o estudo. No pré-parto, houve uma incidência de aproximadamente $50 \%$ no verão e 70\% no inverno (Figura 4). Estes valores caíram com a aproximação do parto e nos dias logo em seguida, mas voltaram a aumentar a partir dos 10 dias pós-parto.

A condição corporal dos animais era maior no inverno e permaneceu assim durante todo o pré-parto (Quadro 4). No dia do parto a condição corporal nos dois períodos se igualou, demonstrando perda de condição corporal dos animais no inverno durante o pré-parto. Do dia do parto até 30 dias pós-parto houve uma queda de pequena inten- sidade e progressiva na condição corporal dos animais nas duas estações.

\section{DISCUSSÃO}

No presente estudo, apesar de muitos animais apresentarem níveis plasmáticos de cálcio inferiores ao intervalo de referência para a espécie, nenhum animal apresentou o quadro clínico da hipocalcemia. Este resultado está de acordo com Goff \& Horst (1997b) que afirmam que, embora a hipocalcemia clínica apresente baixa incidência, praticamente todas as vacas experimentam alguma redução no cálcio sanguíneo durante os primeiros dias após o parto, enquanto se adapta às demandas da lactação.

A média geral de animais hipocalcêmicos no dia do parto neste experimento é de $52,2 \%$, muito próxima dos $50 \%$ descrita na literatura (Reinhardt et al. 1988, Goff \& Horst 1997b, Goff 2008, Goff 2009, Oetzel 2013).

0 manejo da propriedade teve grande influência nos resultados encontrados. 0 fornecimento da dieta para as vacas no pré-parto possuía algumas deficiências, como espaço de cocho reduzido para o grande número de animais e não utilização de estratégias nutricionais para a prevenção de hipocalcemia. Esses problemas acabaram afetando mais os animais no período de inverno, quando toda a dieta era fornecida no cocho e o pasto se apresentava com baixa qualidade e disponibilidade. No verão, o pasto possuía grande 
disponibilidade de forragem e alta qualidade, permitindo que os animais conseguissem suprir suas exigências, refletindo em uma melhor homeostase mineral em relação aos animais no inverno. A influência da disponibilidade de forragem também fica evidente pela diferença da condição corporal nas duas estações do ano (Quadro 4). Observou-se que os animais no inverno possuíam maior condição corporal média quatro semanas pré-parto, porém apresentaram queda mais acentuada até o parto do que os animais do verão. Dessa forma, as menores concentrações de cálcio e magnésio observadas no inverno podem estar relacionadas, em parte, a uma menor ingestão de alimentos devido a fatores de manejo da propriedade.

A queda dos níveis de cálcio próximo ao parto possivelmente está relacionada com o balanço negativo gerado pela grande demanda desse mineral para produção de colostro e leite, diminuição da ingestão de alimentos e fase de adaptação dos mecanismos de homeostase de cálcio (Goff \& Horst 1997a e b, Goff 2004, DeGaris \& Lean 2008).

A redução nos valores da concentração de cálcio no dia do parto foi similar às observações feitas por alguns autores que verificaram redução do mineral com a aproximação do parto e no dia do parto (Blum et al. 1972, Wilson et al. 1977, Van Mosel \& Corlett 1990, Van Mosel et al. 1991, Vanden Top et al. 1995, Goff \& Horst 1997a, Souza Júnior et al. 2011, Buture 2009).

No Brasil, os estudos existentes corroboraram com os achados desse experimento (Buture 2009, De Paula et al. 2011, Souza Júnior et al. 2011). Nos trabalhos de Souza Júnior et al. (2011) e De Paula (2011) as concentrações médias de cálcio no dia do parto $(7,69 \mathrm{mg} / \mathrm{dL}$ e $7,77 \pm 1,0 \mathrm{mg} /$ $\mathrm{dL}$ respectivamente) foram mais baixas que as encontradas nesse experimento $(8,7 \mathrm{mg} / \mathrm{dL})$. As diferenças encontradas entre estes trabalhos ressaltam a importância da implementação do monitoramento do período periparto de cada propriedade.

No presente estudo, as concentrações médias de cálcio voltaram aos níveis encontrados no pré-parto depois de aproximadamente dez dias em lactação. Esta tendência foi observada por Van den Top et al. (1995), Wu et al. (2000), Busato (2002), Souza Júnior (2008) e De Paula et al. (2011), demonstrando que nesse período, o animal ainda está passando por um processo de adaptação as novas demandas desse mineral.

A incidência de hipocalcemia subclínica também segue esse comportamento com os maiores níveis ocorrendo até os 10 primeiros dias pós-parto, com aproximadamente $50 \%$ de animais apresentando hipocalcemia subclínica no inverno e $25 \%$ no verão.

Isso demonstra que o período imediatamente antes do parto até os dez dias pós-parto apresenta o maior desafio a homeostase do cálcio e que com o decorrer da lactação o organismo consegue se adequar as demandas desse mineral e mantém seus níveis plasmáticos adequados.

Outro importante resultado foi a grande incidência de animais com hipocalcemia subclínica por um longo período no inverno, sendo que aos 30 dias, ainda existia uma incidência de hipocalcemia subclínica de 23,3\%.

A taxa de $35,48 \%$ de incidência de hipocalcemia sub- clínica encontrada no dia do parto no verão está próxima da taxa de 30\% considerada desejável por Oetzel (2004) e Mulligan et al. (2006), porém a taxa de $75 \%$ no inverno está muito acima.

Os resultados descritos indicam que os animais no inverno tiveram um declínio mais acentuado nas concentrações de cálcio e que permaneceram mais tempo em níveis que prejudicam a saúde e produtividade do animal.

A alta incidência e duração da hipocalcemia subclínica principalmente no inverno são de extrema relevância uma vez que a mesma contribui para inapetência em vacas recém-paridas, exacerba a imunossupressão experimentada durante o período de transição, predispõe o animal ao desenvolvimento de cetose, retenção de placenta, deslocamento de abomaso, mastite, metrite, distocias e, como consequência, diminui a vida produtiva do animal (Curtis et al. 1983, Oetzel 1988, 2013, Goff \& Horst 1997b, NRC 2001, Goff 2004, Kimura et al. 2006, Goff 2008, Martinez et al. 2012).

A homeostase do cálcio é regulada pela a calcitonina, pelo paratormônio (PTH) e pela 1,25-dihidroxivitamina D (Littledike \& Goff 1987, DeGaris \& Lean 2008). Quando as concentrações de cálcio são menores que o intervalo de referência, como observada nos animais próximo ao parto, ocorre à liberação do paratormônio, que aumenta a reabsorção óssea e ativa a 1,25-dihidroxivitamina D. A vitamina D atua aumentando a reabsorção tubular renal e a absorção intestinal de cálcio (DeGaris \& Lean 2008, Taylor et al. 2008, Goff 2009). Porém, em algumas situações esses mecanismos falham e ocorre a hipocalcemia (NRC 2001).

Normalmente, as concentrações de paratormônio e de 1,25- dihidroxivitamina D aumentam nas primeiras 24 horas pós-parto (Goff \& Horst 1997a) sendo que, na maior parte das vezes, essa não é a causa determinante para a ocorrência de hipocalcemia. 0 grande problema é a demora de resposta de alguns mecanismos, principalmente a reabsorção óssea que necessita de algum tempo para ser ativada e fatores que causam resistência dos receptores ou que interferem na resposta aos hormônios (Curtis et al. 1983, Oetzel 1988, Goff \& Horst 1997a, NRC 2001, DeGaris \& Lean 2008, Goff 2008).

Vários são os fatores que podem interferir na homeostase do cálcio, entre eles figuram como principais a composição da dieta, idade, sexo e raça (Reinhardt et al. 1988).

A diferença apresentada nas concentrações de cálcio entre os dois períodos se deve, entre outras causas, à diferença na dieta entre os dois períodos. As concentrações de magnésio, fósforo e potássio na dieta são os principais fatores que podem interferir na homeostase do cálcio. Levando em consideração essas interações e analisando os resultados de cálcio, magnésio e fósforo concomitantemente, podemos concluir que a maior ocorrência de hipomagnesemia e hiperfosfatemia no inverno foram fatores que contribuíram para a diferença encontrada nas concentrações de cálcio e na incidência de hipocalcemia entre os animais no verão e no inverno.

Os valores médios de fósforo sérico encontrados nesse estudo variaram entre 9,02 e $6,95 \mathrm{mg} / \mathrm{dL}$ no verão e entre 9,11 e $7,64 \mathrm{mg} / \mathrm{dL}$ no inverno, estando em alguns mo- 
mentos, principalmente durante o pré-parto, acima dos intervalos fisiológicos de 4 a $8 \mathrm{mg} / \mathrm{dL}$ (Goff 2000). Apesar de somente no dia 15 pós-parto ter havido diferença nas médias de fósforo entre os dois períodos, analisando a incidência de hiperfosfatemia constatamos maior ocorrência no inverno (média geral de 37,3\% no verão versus 49,8\% no inverno). A hiperfosfatemia é capaz de interferir na conversão renal da vitamina D em 1,25-dihidroxivitamina D, diminuindo sua concentração e prejudicando a absorção intestinal de cálcio (Goff 2004, DeGaris \& Lean 2008).

No dia do parto, as baixas concentrações de cálcio sérico, provavelmente elevaram as concentrações de paratormônio como resposta. 0 paratormônio promove a perda renal e salivar de fósforo, acarretando a diminuição da sua concentração sérica (Goff 2000, 2006). Esse foi um provável motivo pelo qual as concentrações de fósforo sofreram redução próxima ao parto, estando esse achado de acordo com Blum et al. (1972), Wilson et al.(1977), Reinhardt et al. (1988) e Van Mosel et al. (1991).

A produção de colostro e o crescimento fetal consomem grande quantidade de fósforo (Goff 2006), colaborando com a diminuição das concentrações desse mineral observada nas semanas imediatamente antes do parto.

No Brasil, Buture (2009) também encontrou queda nos níveis de fósforo no dia do parto com posterior elevação em vacas leiteiras em sistema semi-intensivo no estado do Paraná, porém De Paula et al. (2011) não observou diferença nas concentrações de fósforo no peri-parto de vacas em sistema semi-intensivo. Souza Júnior et al. (2011) relata variações nas concentrações de fósforo em coletas feitas com intervalos de horas depois do parto, encontrando concentrações menores entre duas e seis horas subsequentes ao parto, refletindo o dinamismo da homeostase mineral.

As concentrações de fósforo no pós-parto foram, em quase todos os momentos, menores que as concentrações no pré-parto. Durante a lactação a vaca necessita de mobilizar cerca de um grama de fósforo da reserva extracelular para a produção de um quilograma de leite (Goff 2006).

A grande quantidade de animais com hiperfosfatemia está relacionada com uma suplementação maior do que a necessária, pois os níveis plasmáticos de fósforo são muito dependentes da sua ingestão e absorção (Reinhardt et al. 1988). Existe uma tendência de suplementar fósforo em excesso devido ao medo de diminuir o desempenho produtivo dos animais (Wu et al. 2000, Malafaia et al. 2014).

Outro fator que deve ter contribuído para os resultados do cálcio no inverno foi a alta incidência de hipomagnesemia. Esta interfere na homeostase do cálcio, pois o paratormônio ativa a adenilciclase ao se ligar ao seu receptor nos ossos e no rim, ou ativa a fosfolipase $\mathrm{C}$ quando se liga a receptores em outros tecidos. Ambos adenilciclase e fosfolipase $\mathrm{C}$ possuem um sítio de ligação com o íon $\mathrm{Mg}^{2+}$, o qual deve estar ocupado para que haja total resposta ao hormônio (Goff 2004, 2008).

Levando em consideração que o magnésio não possui uma regulação própria e que os seus níveis estão muito relacionados com a absorção da dieta (Chester-Jones et al. 1989, Vormann 2003), uma diferença mesmo que pequena entre as dietas pode ser o motivo da variação na concentra- ção de magnésio entre as duas estações. Normalmente, o pasto é uma ótima fonte de magnésio (Castro et al. 2009), e sua inclusão na dieta dos animais no verão pode ter auxiliado para que esses tivessem uma melhor homeostase desse mineral.

A queda nas concentrações de magnésio após o parto também foi descrita por Wilson et al. (1977) e Van den Top et al. (1995), os quais mostraram níveis mais baixos de magnésio 48 horas após o parto e na semana seguinte ao parto respectivamente. Van den Top et al. (1995) relataram que as concentrações de magnésio retornaram a níveis semelhantes aos do pré-parto na quarta semana pós-parto.

Durante a lactação, os níveis séricos de magnésio podem sofrer uma queda devido à excreção deste como componente do leite e assim, sua exigência aumenta com o início da lactação. A glândula mamária necessita de cerca de $120 \mathrm{mg}$ de magnésio para produção de um quilograma de leite, sendo que altas produções podem exigir três gramas por dia, o que representa grande quantidade do magnésio absorvido (Robertson et al. 1960, Dalley 1992). Leaver (1972) descreveu que devido à alta secreção de magnésio no leite e dietas deficientes, a mobilização de reservas corporais não consegue manter os níveis normais, e por isso sua concentração plasmática declina nessas situações.

Resultados diferentes do presente estudo são relatados por Field (1970) que encontrou um aumento do magnésio com o aumento do tempo de lactação, e Aeberhard et al. (2001) que encontraram concentrações de magnésio no pós-parto maiores que as concentrações pré-parto.

No Brasil os estudos são escassos, sendo que Souza Júnior (2008) e Buture (2009) não relataram variação do magnésio antes do parto, no parto e nos dias após o parto. Souza Júnior et al. (2011), encontraram níveis séricos de magnésio mais baixos do que os encontrados no presente estudo e muito próximos dos níveis de limiar renal de 1,8mg/dL (Dalley 1992). Quando a concentração de magnésio é inferior a esse limite, a reabsorção renal aumenta muito e, por esse motivo, os níveis de magnésio podem não ter sofrido variações no estudo de Souza Júnior (2011) devido a esse mecanismo de controle.

No dia do parto, as concentrações de magnésio foram similares às do pré-parto, porém, alguns autores relatam elevação nas concentrações de magnésio nesse momento (Marquardt et al. 1976, Wilson et al. 1977, Larsen 2001, Radostits et al. 2007), o que poderia ser justificado pela baixa concentração de cálcio no parto que estimula a liberação do paratormônio. Este, por sua vez, aumenta o limiar renal para excreção de magnésio e estimula a reabsorção óssea, promovendo o aumento da concentração plasmática de magnésio (Dalley 1992, Larsen 2001). Similar ao observado neste estudo, Souza Júnior (2008) não encontrou alterações nos níveis plasmáticos de magnésio no dia do parto. Em outro estudo brasileiro, De Paula et al. (2011) trabalhando com vacas em sistema semi-intensivo, relataram diminuição das concentrações de magnésio no dia do parto, enquanto Aquino Neto (2012) encontrou aumento dos níveis de magnésio com dois dias após o parto em vacas confinadas.

O motivo que fez o magnésio não variar no dia do parto 
neste estudo, foi o equilíbrio entre a utilização deste mineral para a produção de colostro e a reposição deste através da dieta e também pela absorção óssea e diminuição da excreção renal pela ação do paratormônio. Como existiam muitas vacas hipocalcêmicas neste momento $(35,48 \%$ no verão e $75 \%$ no inverno) o paratormônio provavelmente estava aumentado, favorecendo o aumento ou mantença das concentrações séricas de magnésio.

As grandes variações entre os resultados dos vários trabalhos em relação ao comportamento e aos valores de magnésio ocorrem pela falta de mecanismos regulatórios da concentração deste mineral no soro (NRC 2001, Goff 2004), recaindo sobre a dieta fornecida a maior parte da responsabilidade pelos valores encontrados.

\section{CONCLUSÕES}

A variação nas concentrações médias dos minerais no peri-parto demonstra que ocorreu uma readaptação do organismo durante o período de transição para conseguir manter a homeostase dos minerais.

A homeostase mineral foi mais eficiente no período de verão, quando os animais conseguiram se adequar mais rapidamente e com menores transtornos às novas exigências nutricionais, enquanto as vacas no inverno tiveram menores concentrações de cálcio e magnésio refletindo em uma maior incidência de hipocalcemia e hipomagnesemia, com uma longa duração destes episódios.

0 perfil mineral de vacas mestiças em sistema semi-intensivo demonstra significativa diferença entre verão e inverno, motivada pelas alterações de manejo e alimentação oferecidas aos animais. Isso indica que fatores de manejo influenciam de forma marcante os mecanismos de homeostase mineral.

O perfil mineral e as incidências das desordens metabólicas nas duas estações do ano são ferramentas importantes como monitoramento da saúde e produtividade do rebanho leiteiro e funcionam como parâmetros de avaliação dos manejos utilizados.

A avaliação do perfil mineral deve ser realizada em cada propriedade devido a grande variação observada na literatura mesmo em propriedades com manejos similares devido ao grande número de fatores que podem interferir nos mecanismos de homeostase.

\section{REFERÊNCIAS}

Aeberhard K., Bruckmaier R.M. \& Blum J.W. 2001. Metabolic, enzymatic and endocrine status in high yielding dairy cows - Part 2. J. Vet. Med. A, Physiol. Pathol. Clin. Med. 48:111-127.

Aquino Neto H.M.A. 2012. Perfil hidroeletrolítico, ácido-base, metabólico e mineral de vacas leiteiras no pós-parto imediato e avaliação da fluidoterapia oral. Tese de Doutorado, Universidade Federal de Minas Gerais, Belo Horizonte, MG. 121p.

Blum J.W., Ramberg C.F., Johnson K.G. \& Kronfeld D.S. 1972. Calcium (ionized and total), magnesium, phosphorus, and glucose in plasma from parturient cows. Am. J. Vet. Res. 33:51-56.

Busato A., Faissle D., Kupfer U. \& Blum J.W. 2002. Body condition scores in dairy cows: associations with metabolic and endocrine changes in healthy dairy cows. J. Vet. Med. A, Physiol. Pathol. Clin. Med. 49:455-460.

Buture I.O. 2009. Avaliação metabólica de bovinos leiteiros no periparto como forma de diagnóstico precoce da hipocalcemia da vaca leiteira.
Tese de Doutorado em Ciência Animal, Universidade Estadual de Londrina, Londrina, PR. 173p.

Castro G.H.F., Martins R.G.R., Gonçalves L.C. \& Coelho S.G. 2009. Minerais na nutrição de bovinos de leite, p.304-338. In: Gonçalves L.C., Borges I. \& Ferreira P.D.S. (Eds), Alimentação de Gado de leite. Editora FEPMVZ, Belo Horizonte.

Chapinal N., Carson M., Duffield T.F., Capel M., Godden S., Overton M., Santos J.E. \& LeBlanc S.L. 2011. The association of serum metabolites with clinical disease during the transition period. J. Dairy Sci. 94:4897-4903.

Chester-Jones H., Fontenot J.P., Veit H.P. \& Webb K.E. 1989. Physiological effects of feeding high levels of magnesium to sheep. J. Anim. Sci. 67:1070-1081.

Curtis C.R., Erb H.N. \& Sniffen C.J. 1983. Association of parturient hypocalcemia with eight periparturient disorders in Holstein cows. J. Am. Vet. Med. Assoc. 183:559-561.

Dalley D.E. 1992. Studies of magnesium metabolism in ruminants. Tese de Doutorado em Filosofia, Lincoln University, Christchurch, N.Z. 149p.

De Paula V.M., Freitas M.D., Moreira T.F., Moreira G.H.F.A., Ferreira L.O., Salgado L.M., Leme F.O.P., Molina L.R., Carvalho A.U. \& Facury Filho E.J. 2011. Perfil mineral e bioquímico de vacas leiteiras no período de transição em um sistema semi-intensivo em Minas Gerais. Anais 9o Congresso Brasileiro de Buiatria, Goiânia, GO, p.650-654.

DeGaris P.J. \& Lean I.J. 2008. Milk fever in dairy cows: A review of pathophysiology and control principles. Vet. J. 176:58-69.

Drackley J.K., Dann H.M., Douglas G.N., Guretzky N.A.J., Litherland N.B., Underwood J.P. \& Loor J.L. 2005. Physiological and pathological adaptations in dairy cows that may increase susceptibility to periparturient diseases and disorders. Ital. J. Anim. Sci. 4:323-344.

Field A.C. 1970. Studies on magnesium in ruminant nutrition.Effect of lactation on the excretion of magnesium and faecal dry matter by grazing monozygotic twin cows. Brit. J. Nutr. 24:71-83.

Froetschel M.A. 2011. Nutritional intervention to improve the calcium and energetic status of high producing transition cows. Proc. 25th Annual Meeting of the Southeast Dairy Herd Management Conference, University of Georgia, Georgia, p.19-27.

Goff J.P. \& Horst R.L. 1997a. Effects of the addition of potassium or sodium, but not calcium, to prepartum rations on milk fever in dairy cows. J. Dairy Sci. 80(1):176-186.

Goff J.P. \& Horst R.L. 1997b. Physiological changes at parturition and their relationship to metabolic disorders. J. Dairy Sci. 80(7):1260-1267.

Goff J.P. 2000. Pathophysiology of calcium and phosphorus disorders. In Metabolic Diseases of Ruminant Livestock. Vet. Clin. North Am., Food Anim. Pract. 16:319-337.

Goff J.P. 2004. Macromineral disorders of the transition cow. Vet. Clin. North Am., Food Anim. Pract. 20(3):471-494.

Goff J.P. 2006. Macromineral physiology and application to the feeding of the dairy cow for prevention of milk fever and other periparturient mineral disorders. Anim. Feed Sci. Technol. 126:237-257.

Goff J.P. 2008. The monitoring, prevention, and treatment of milk fever and subclinical hypocalcemia in dairy cows. Vet. J. 176:50-57.

Goff J.P. 2009. Como controlar a febre do leite e outras desordens metabólicas relacionadas à macro minerais em vacas de leite. Anais XIII Curso Novos Enfoques na Produção e Reprodução de Bovinos, Uberlândia, MG, p.267-284.

Horst R.L., Goff J.P., Reinhardt T.A. \& Buxton D.R. 1997. Strategies for preventing milk fever in dairy cattle. J. Dairy Sci. 80:1269-1280.

Kimura K., Reinhardt T.A. \& Goff J.P. 2006. Parturition and hypocalcemia blunts calcium signals in immune cells of dairy cattle. J. Dairy Sci. 89:2588-2595.

Larsen T., Møller G. \& Bellio R. 2001. Evaluation of clinical and clinical chemical parameters in periparturient cows. J. Dairy Sci. 84:1749-1758.

Leaver D.D. 1972. Grass tetany of cattle and sheep. In: Aust. Meat Research Committee Reviews, n.8, Sydney.

Littledike E.T. \& Goff J. 1987. Interactions of calcium, phosphorus, magnesium and vitamin $d$ that influence their status in domestic meat animals. J. Anim. Sci. 65:1727-1743. 
Malafaia P., Magnoli Costa R., Brito M.F., Peixoto P.V., Barbosa J.D., Tokarnia C.H. \& Döbereiner J. 2014. Equívocos arraigados no meio pecuário sobre deficiências e suplementação minerais em bovinos no Brasil. Pesq. Vet. Bras. 34(3)244-249.

Marquardt J.P., Horst R.L. \& Jorgensen N.A. 1976. Effect of parity on dry matter intake at parturition in dairy cattle. J. Dairy Sci. 60(6):929-934.

Martinez N., Risco C.A., Lima F.S., Bisinotto R.S., Greco L.F., Ribeiro E.S., Maunsell F., Galvão K. \& Santos J.E.P. 2012. Evaluation of peripartal calcium status, energetic profile, and neutrophil function in dairy cows at low or high risk of developing uterine disease. J. Dairy Sci. 95:1-15.

Melendez P., Donovan A., Risco C. \& Goff J.P. 2004. Plasma mineral and energy metabolite concentrations in dairy cows fed an anionic prepartum diet that did or did not have retained fetal membranes after parturition. Am. J. Vet. Res. 65:1071-1076.

Mulligan F. J., O'Grady L., Rice D.A. \& Doherty M.L. 2006. A herd health approach to dairy cow nutrition and production diseases of the transition cow. Anim. Reprod. Sci. 96:331-353.

NRC 2001. Nutritional Requirements of Dairy Cattle. National Research Council, Washington, D.C. National Academy Press. 370p.

Oetzel G.R. \& Goff J.P. 2009. Milk fever (parturiente paresis) in cows, ewes, and doe goats, p.130-134. In: Anderson D.E. \& Rings D.M. (Eds), Current Veterinary Therapy: Food Animal Practice. 5th ed. Saunders Elsevier, St Louis.

Oetzel G.R. 1988. Parturient paresis and hypocalcemia in ruminant livestock. In: Metabolic Diseases of Ruminant Livestock. Vet. Clin. North Am., Food Anim. Pract. 4(2):351-364.

Oetzel G.R. 2004. Monitoring and testing dairy herds for metabolic disease. Vet. Clin.North Am., Food Anim. Pract. 20:651-674.

Oetzel G.R. 2013. Oral calcium supplementation in peripartum dairy cows. In: Metabolic Diseases of Dairy Cattle. Vet. Clin. North Am. Food Anim. Pract. 29:447-455.

Payne J.M., Dew S.M., Mansto R. \& Faulks M. 1970. The use of a metabolic profile test in dairy herds. Vet. Rec. 87:150-158.

Radostits O.M., Gay C.C., Hinchcliff K.W. \& Blood D.C. 2007. Veterinary Medicine: a textbook of the diseases of cattle, horses, sheep, pigs and goats. 10th ed. Elsevier, Philadelphia. 2156p.

Reinhardt T.A., Horst R.L. \& Goff J.P. 1988. Calcium, phosphorus and magnesium homeostasis in ruminants. In: Metabolic Diseases of Ruminant Livestock. Vet. Clin. North Am., Food Anim. Pract. 4(2):331-350.

Reinhardt T.A., Lippolis J.D., McCluskey B.J., Goff J.P. \& Horst R.L. 2011. Prevalence of subclinical hypocalcemia in dairy herds. Vet. J. 188:122-124.

Roberts T., Chapinal N., LeBlanc S.J., Kelton D.F., Dubuc J. \& Duffield T.F. 2012. Metabolic parameters in transition cows as indicators for earlylactation culling risk. J. Dairy Sci. 95(6):3057-3063.
Robertson A., Paver H., Barden P. \& Marr T.G. 1960. Fasting metabolism of the lactating cow. Res. Vet. Sci. 1:117-124.

Sampaio I.B.M. 2010. Estatística Aplicada à Experimentação Animal. 3aㅡ ed. Editora FEPMVZ, Belo Horizonte 265p.

Souza Júnior J.A. 2008. Influência da adição de monensina e propilenoglicol na dieta do periparto de vacas leiteiras sobre o comportamento das concentrações de cálcio, fósforo, magnésio, sódio e potássio. Dissertação de Mestrado em Ciência Animal, Universidade Federal de Minas Gerais, Belo Horizonte, MG. 88p.

Souza Júnior J.A., Moreira T.F., Lasmar P.V.F., Ferreira L.O., Aquino Neto H.M., Freitas M.D., Zambrano J.A., Molina L.R., Carvalho A.U. \& Facury Filho E.J. 2011. Efeito da adição de monensina ou propilenoglicol na dieta de vacas leiteiras no periparto sobre concentrações séricas de minerais. Anais 9o Congresso Brasileiro de Buiatria, Goiânia, GO, p.636-639.

Stengärde L. 2010. Displaced abomasum and ketosis in dairy cows blood profiles and risk factors. Tese de Doutorado em Medicina Veterinária, Swedish University of Agricultural Sciences, Uppsala, Suécia. 76p.

Taylor M.S., Knowton K.F., McGilliard M.L., Seymour W.M. \& Herbein J.H. 2008. Blood mineral, hormone, and osteocalcin responses of multiparous jersey cows to an oral dose of 25-hydroxyvitamin D3 or vitamin D3 before parturition. J. Dairy Sci. 91(6):2408-2416.

Van Den Top A.M., Wensing T. \& Beynen A.C. 1995. Influence of replacement of dietary hay by an isoenergetic amount of a concentrate rich in medium chain triacylglycerols on fat metabolism in goats. J. Anim. Physiol. Anim. Nutr. 73:104-112.

Van Mosel M. \& Corlett S.C. 1990. Assessment of bone turnover in the dry period of dairy cows by measurement of plasma bone gla protein, total plasma alkaline phosphatase activity and urinary hydroxyproline. Exp. Physiol. 75:827-837.

Van Mosel M., Vant Klooster A.T.H. \& Wouterse H.S. 1991. Effects of deficient magnesium supply during the dry period on bone turnover of dairy cows at parturition. Vet. Quart. 13:199-209.

Vormann J. 2003. Magnesium: nutrition and metabolism. Mol. Asp. Med. 24:27-37.

Whitaker D.A. 2004. Metabolic profiles, p.804-817. In: Andrews A.H., Blowey R.W., Boyd H. \& Eddy R.G. (Eds), Bovine Medicine: Diseases and Husbandry of Cattle. 2nd ed. Wiley-Blackwell, Oxford.

Whitaker D.A., Goodger W.J., Garcia M., Pereira B.M.A.O. \& Wittwer F. 1999. Use of metabolic profiles in dairy cattle in tropical and subtropical countries on smallholder dairy farms. Prev. Vet. Med.38:119-131.

Wilson G.D.A., Hunter J.T., Derrick G.H., Aitken W.M. \& Kronfeld D.S. 1977. Fetal and maternal mineral concentrations in dairy cattle during late pregnancy. J. Dairy Sci. 60(6):935-941.

Wu Z., Satter L.D. \& Sojo R. 2000. Milk production, reproductive performance, and fecal excretion of phosphorus by dairy cows fed three amounts of phosphorus. J. Dairy Sci. 83:1028-1041. 